See discussions, stats, and author profiles for this publication at: https://www.researchgate.net/publication/337242957

CÁC YẾU TỐ TÁC ĐỘNG ĐẾN QUYẾT ĐỊNH SỬ DỤNG DỊCH VỤ NGÂN HÀNG ĐIỆN TỬ CỦA KHÁCH HÀNG CÁ NHÂN TẠI NGÂN HÀNG AGRIBANK - CHI NHÁNH CẦN THO

Article $\cdot$ November 2019

1 author:

Le Chau Phu

1 PUBLICATION 0 CITATIONS

SEE PROFILE 


\title{
CÁC YẾU TỐ TÁC ĐộNG ĐẾN QUYẾT ĐỊNH SỬ DỤNG DỊCH VỤ NGÂN HÀNG ĐIỆN TỬ CỦA KHÁĊH HÀNG CÁ NHÂN TẠI NGẦ HÀNG AGṘBBANK - CHI NHÁNH CẦN THƠ
}

\section{TÓM TẮT}

Ths. Lê Châu Phú, PGS. TS. Đào Duy Huân

Mục tiêu của nghiên cứu này là để khám phá ra yếu tố chính tác động đến quyết định sử dụng dịch vụ ngân hàng điện tử của khách hàng cá nhân tại Ngân hàng Agribank Cần Thơ. Dữ liệu khảo soát đã thu thập từ 340 khách hàng cá nhân đã đăng ký sử dụng dịch vụ ngân hàng điện tử tại ngân hàng Agribank Cần Thơ đã được phân tích để cung cấp bằng chứng. Kết quả từ phân tích hồi quy bội bởi sử dụng phần mềm SPSS đã tiết lộ rằng có 6 yếu tố và mức độ tác động giảm dần đến quyết định sử dụng dịch vụ ngân hàng điện tử của khách hàng cá nhân tại Agribank Cần Thơ như là: Hiệu quả mong đợi; Rủi ro trong giao dịch; Cảm nhận dễ sử dụng; Sự ưa thích cảm nhận; Ảnh hưởng xã hội; Thương hiệu ngân hàng. Từ kết quả đó, tác giả đưa ra các hàm ý quản trị nhằm thu hút khách hàng cá nhân sử dụng dịch vụ Ngân hàng điện tử tại Agribank Cần Tho.

Tù khóa: hiệu quả mong đọi, rủi ro trong giao dịch, cảm nhận dễ sủ̉ dụng; sụ̂ ưa thích cảm nhận, Anh hwởng xã hội, Thuơng hiệu ngân hàng.

\begin{abstract}
The objective of this study is to discover the key factors influencing individual customers' decision to use e-banking services at Agribank in Can Tho branch. Survey data collected from 340 individual customers who registered to use e-banking services at Agribank in Can Tho branch were analyzed to provide evidence. Results from multiple regression analysis by using SPSS software have revealed that there are 6 factors impact on the decision to use e-banking services of individual customers at Agribank in Can Tho such as (1) Expected efficiency; (2) Perceived risk in transactions; (3) Perceived ease to use; (4) Perceived enjoyment (5) Social influence; (6) Bank brand. From that result, the author gives management implications to attract individual customers to use E-banking services at Agribank in Can Tho branch.
\end{abstract}

Keywords: expected efficiency, perceived risk in transactions, perceived ease to use, perceived enjoyment, social influence, bank brand

\section{GIỚI THIẸU}

Trong những năm qua cùng với sự phát triển nhanh chóng về công nghệ thông tin nói chung, dịch vụ ngân hàng điện tử cũng đã có những bước tiến vượt bậc. Bên cạnh các dịch vụ ngân hàng truyền thống thì dịch vụ ngân hàng điện tử đã và đang góp phần làm đa dạng hóa các loại hình dịch vụ của hệ thống ngân hàng thương mại và là dịch vụ không thể thiếu trong quá trình hội nhập quốc tế.

Hiện nay, với sự bùng nổ của cuộc cách mạng 4.0, sự gia tăng số lượng người sử dụng mạng internet đã tạo ra vô vàng các cơ hội cho các tổ chức, bao gồm cả các tổ chức tài chính. Đặc biệt ngân hàng và các tổ chức tài chính đã thực thi công cụ internet nhằm nắm giữ thị phần. Ngân hàng điện tử sử dụng công cụ internet như là một kênh để cung cấp các dịch vụ của ngân hàng như là xem số dư, sao kê tài khoản, chuyển tiền, thanh toán hóa đơn. Xét từ gốc độ phía ngân hàng, ngân hàng điện tử sẽ giúp ngân hàng phát triển lợi nhuận thông qua giảm chi phí hoạt động và chi phí cố định. Theo Jayawardhena và Foley (2000) chi phí một giao dịch đơn giản không liên quan đến tiền mặt tại ngân hàng thì gấp 11 lần giao dịch tương tự thông qua ngân hàng điện tử. Mặt khác, theo hai tác giả này, thông qua internet ngân hàng có thể nâng cao tiếp thị và thông tin bởi vì thời gian hoạt động của nó không bị giới hạn như khi giao dịch 
tại ngân hàng, đối với dịch vụ này khách hàng có thể giao dịch 24/24 và có thể được hướng dẫn thông qua bảng chỉ dẫn sử dụng dịch vụ do ngân hàng cung cấp. Xét từ khía cạnh khách hàng, dịch vụ ngân hàng điện tử mang lợi sự thuận tiện với mức phí hấp dẫn. Song song đó khách hàng có thể thực hiện các giao dịch ở bất cứ thời gian và địa điểm nào mà không phải tốn thời gian chờ đợi tại ngân hàng.

Thanh toán trực tuyến qua ngân hàng điện tử (internet banking, mobile banking, web banking) ở Việt Nam chỉ mới phát triển vài năm gần đây nhưng đang thật sự bùng nổ khi lượng người dùng mua sắm hàng hóa, dịch vụ qua các ứng dụng này ngày càng tăng. Việt Nam với dân số 92 triệu người có đến $55 \%$ người sử dụng smartphone và truy cập mạng bình quân 2 giờ mỗi ngày. Mua sắm trực tuyến chiếm tỉ lệ $58 \%$. Tốc độ tăng trưởng mạnh mẽ của thị trường thẻ ngân hàng cũng cho thấy xu hướng tiêu dùng không bằng tiền mặt, góp phần phát triển các dịch vụ thanh toán trên di động. Đến năm 2018, có khoảng 45 ngân hàng cung cấp dịch vụ SMS banking, Internet Banking và 32 ngân hàng phát triển ứng dụng internet banking. Do đó, đứng trước một thị trường cạnh tranh với nhiều ngân hàng thương mại, thu hút khách hàng nhờ khoa học công nghệ hiện đại như Techcombank, Á Châu, Vietcombank....ngân hàng Agribank Cần Thơ không thể chỉ biết dựa vào các sản phẩm truyền thống mà phải biết đi theo cái nhìn của thời đại. Nắm được xu thế này, ngân hàng đã không ngừng đổi mới, cải tiến các sản phẩm dịch vụ của mình để thu hút khách hàng. Tuy nhiên, dịch vụ ngân hàng điện tử mới chỉ được Agribank Cần Thơ triển khai trong những năm gần đây. Để có thế nhanh chóng đưa dịch vụ ngân hàng điện tử đến với đại đa số khách hàng cá nhân của Agribank Cần Thơ cần phải xác định các yếu tố đóng vai trò quyết định đến việc sử dụng dịch vụ ngân hàng điện tử của khách hàng.

\section{CƠ SỞ LÝ THUYẾT}

\section{Ngân hàng điện tử}

Ngân hàng điện tử là hình thức thực hiện các giao dịch tài chính, ngân hàng thông qua các phương tiện điện tử. Hiện nay, Ngân hàng điện tử đã được nhiều ngân hàng sử dụng, cho phép khách hàng có thế thực hiện các giao dịch trực tuyến thông qua các phương tiện như máy vi tính, điện thoại di động hay các thiết bị trợ giúp cá nhân khác...Qua đó, khách hàng có thể truy cập vào mọi thời điểm mà không cần phải đến ngân hàng.

Cùng với sự phát triển của công nghệ và nhu cầu của nền kinh tế, xu thế phát triên của dịch vụ ngân hàng tiến tới ngân hàng điện tử là một xu thế tất yếu. Có rất nhiều cách hiểu khác nhau vể "Ngân hàng điện tử". Song nhìn chung "Ngân hàng điện tử" được hiểu là một loại hình thương mại về tài chính, ngân hàng với sự trợ giúp của công nghệ thông tin. Điều này cho phép khách hàng có thể truy cập từ xa các thông tin, thực hiện các giao dịch thanh toán, tài chính dựa trên các tài khoản lưu ký tại ngân hàng .

\section{Mô hình chấp nhận công nghệ}

Mô hình chấp nhận công nghệ (TAM) được sử dụng để giải thích và dự đoán về sự chấp nhận và sử dụng một công nghệ. TAM được thử nghiệm và chấp nhận một cách rộng rãi trong các nghiên cứu về lĩnh vực công nghệ thông tin, đây được coi là mô hình có giá trị tiên đoán tốt. Trong đó, ý định sử dụng có tương quan đáng kể tới việc sử dụng, khi có ý định là yếu tố quan trọng đến việc sử dụng, còn các yếu tố khác ảnh hưởng đến việc sử dụng một cách gián tiếp thông qua ý định sử dụng.

Biến bên ngoài: là những nhân tố ảnh hưởng đến niềm tin của một người về việc chấp nhận sản phẩm hay dịch vụ. Những biến bên ngoài thường từ hai nguồn là quá trình ảnh hưởng xã hội và quá trình nhận thức, thu thập kinh nghiệm của bản thân (Venkatesh \& Davis, 2000). Sự hữu ích cảm nhận là "Mức độ để một người tin rằng sử dụng hệ thống đặc thù sẽ nâng cao thực hiện công việc của chính họ" (Davis và cộng sự, 1989). Sự dễ sử dụng cảm nhận là "Mức độ mà 
một người tin rằng có thể sử dụng hệ thống đặc thù mà không cần sự nỗ lực" (Davis và cộng sự, 1989). Thái độ là cảm giác tích cực hay tiêu cực về việc thực hiện hành vi mục tiêu (Fishbein \& Ajzen, 1975), đó là nhân tố quan trọng ảnh hưởng tới thành công của hệ thống.

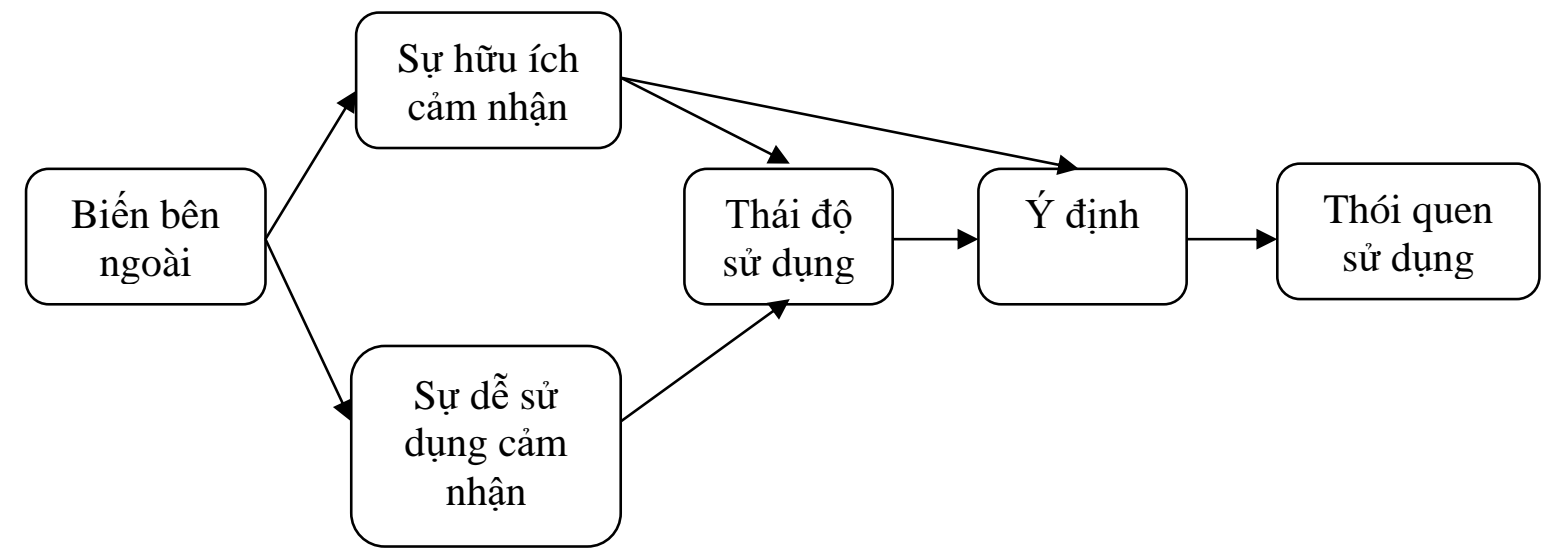

Hình 1. Mô hình chấp nhận công nghệ

Tác giả đã lựa chọn mô hình TAM là cơ sở lý thuyết để xây dựng và phát triển mô hình kiểm định và điều tra các yếu tố tác động đến quyết định lựa chọn dịch vụ ngân hàng điện tử tại Ngân hàng Agribank Cần Thơ.

\section{PHÁT TRIỂN GIẢ THUYẾT NGHIÊN CÚUU}

Nghiên cứu này lựa chọn mô hình dựa vào Thuyết hành động hợp lý của Ajzen và Fishbein (1980), Thuyết hành vi dự định của Ajzen (1991), Mô hình chấp nhận công nghệ của Davis và cộng sự (1989), Thuyết hợp nhất về chấp nhận và sử dụng công nghệ của Venkatesh và các cộng sự (2003). Đây là những mô hình được lựa chọn nghiên cứu trong nhiều lĩnh vực tại nhiều quốc gia châu Á, trong đó có một số nghiên cứu trong lĩnh vực quyết định sử dụng dịch vụ ngân hàng điện tử. Ngoài ra, nghiên cứu dựa vào các nghiên cứu thực nghiệm về quyết định sử dụng dịch vụ ngân hàng điện tử đã được một số tác giả nghiên cứu như: Fatimah và Suyanto (2016), Maditinos và cộng sự (2013). Trên nền tảng cùng là nghiên cứu quyết định sử dụng dịch vụ ngân hàng điện tử, đây sẽ là cơ sở lý thuyết vững chắc, giúp nghiên cứu vận dụng mô hình phù hợp với điều kiện nghiên cứu tại ngân hàng Agribank Cần Thơ. Như vậy, mô hình nghiên cứu đề xuất ban đầu gồm 6 yếu tố: (1) Cảm nhận dễ sử dụng, (2) Hiệu quả mong đợi, (3) Ảnh hưởng xã hội, (4) Rủi ro trong giao dịch, (5) Thương hiệu ngân hàng.

Căn cứ vào kết quả nghiên cứu định tính, $2 / 3$ thành viên trong nhóm thảo luận đồng ý 06 yếu tố mà tác giả đã nêu trong quá trình thảo luận là khá đầy đủ.Bên cạnh đó, nhóm thảo luận đồng ý bổ sung vào 01 yếu tố được đánh giá là có ảnh hưởng đáng kể đối với quyết định sử dụng dịch vụ ngân hàng điện tử, là "Sự ưa thích cảm nhận" vào nghiên cứu tại Agribank Cần Thơ.

$H_{1}$ :Cảm nhận dễ sủ dụng có tác động tích cưc trong quyết định sủ dụng dịch vụ ngân hàng điện tử của khách hàng cá nhân tại Ngân hàng Agribank Cần Tho:

$\mathrm{H}_{2}$ :Hiệu quả mong đợi có tác động tích cực trong quyết định sủ dụng dịch vụ ngân hàng điện tư của khách hàng cá nhân tại Ngân hàng Agribank Cần Tho:

$H_{3}:$ Rủi ro trong giao dịch có tác động tiêu cưcc trong quyết định sủ dụng dịch vu ngân hàng điện tử của khách hàng cá nhân tại Ngân hàng Agribank Cần Tho:

H: Thuoong hiệu ngân hàng có tác động tích cưcc trong quyết định sử dụng dịch vụ ngân hàng điện tử của khách hàng cá nhân tại Ngân hàng Agribank Cần Tho:

H5: Ảnh hưởng xã hội có tác động tích cục trong quyết định sủ dụng dịch vu ngân hàng điện 
tư của khách hàng cá nhân tại Ngân hàng Agribank Cần Tho:

$H_{6}$ : Sự ưa thích cảm nhận có tác động tích cục trong quyết định sủ dụng dịch vụ ngân hàng điện tử của khách hàng cá nhân tại Ngân hàng Agribank Cần Tho.

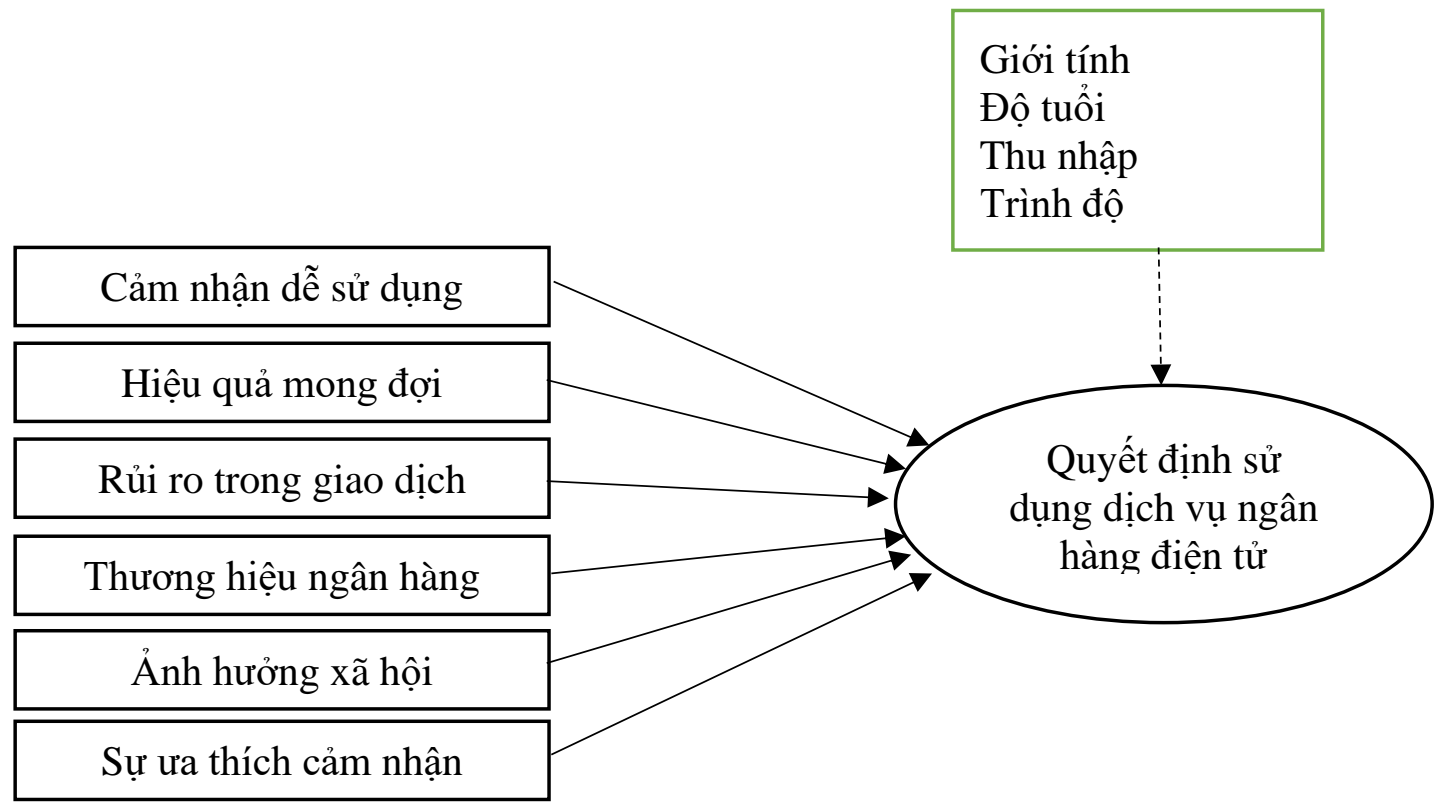

Hình 2. Mô hình nghiên cứu nhóm tác giả đề xuất

\section{KẾT QUẢ NGHIÊN CÚ̉}

\section{Mô tả dữ liệu}

Sau khi kiểm tra 385 bảng câu hỏi được thu thập từ khách hàng thì chỉ còn 340 bảng câu hỏi khảo sát là có câu trả lời đầy đủ và chính xác. Số bảng câu hỏi hợp lệ này được làm dữ liệu cho nghiên cứu. Bảng 1 sẽ trình bày các phân tích thống kê mẫu đã thu thập được.

\section{Bảng 1. Bảng thống kê mô tả đặc điểm khách hàng}

\begin{tabular}{|c|c|c|c|c|}
\hline & & $\begin{array}{c}\text { Tầng } \\
\text { số }\end{array}$ & $\begin{array}{l}\text { Phần } \\
\text { trăm }\end{array}$ & $\begin{array}{c}\text { Phần trăm } \\
\text { tích lũy }\end{array}$ \\
\hline \multirow{2}{*}{ Giới tính } & Nữ & 163 & 47.9 & 47.9 \\
\hline & Nam & 177 & 52.1 & 100.0 \\
\hline \multirow{4}{*}{ Tuổi } & $<22$ tuổi & 93 & 27.4 & 27.4 \\
\hline & Từ 22-35 & 130 & 38.2 & 65.6 \\
\hline & Từ 36-45 & 91 & 26.8 & 92.4 \\
\hline & Trên 45 & 26 & 7.6 & 100.0 \\
\hline \multirow{3}{*}{ Thu nhập } & $<5$ triệu & 8 & 2.4 & 2.4 \\
\hline & $5-<10$ triệu & 115 & 33.8 & 36.2 \\
\hline & $>10$ triệu & 217 & 63.8 & 100.0 \\
\hline \multirow{5}{*}{ Trình độ } & Từ Trung học phổ thông trở xuống & 18 & 5.3 & 5.3 \\
\hline & Trung cấp, Cao đẳng & 102 & 30.0 & 35.3 \\
\hline & Đại học & 209 & 61.5 & 96.8 \\
\hline & Sau đại học & 11 & 3.2 & 100.0 \\
\hline & Tổng & 340 & 100.0 & \\
\hline
\end{tabular}

\section{Đánh giá độ tin cậy của thang đo}

Kiểm định độ tin cậy của thang đo thông qua hệ số Cronbach's alpha. Các biến quan sát có hệ số tương quan biến tổng nhỏ hơn 0,3 sẽ bị loại (Hà Nam Khánh Giao và Bùi Nhất Vương, 
2019). Sau khi phân tích Cronbach's alpha lần 1, biến THNH 4 bị loại do có tương quan với biến tổng nhỏ hơn 0.3 . Kết quả kiểm định lần 2 , các thang đo trên đều có hệ số Cronbach's Alpha khá cao (>0,7), hệ số tương quan biến tổng lớn hơn 0,3 (bảng 2), do đó, được sử dụng cho phân tích EFA tiếp theo.

Bảng 2. Kết quả phân tích độ tin cậy bằng hệ số Cronbach's alpha

\begin{tabular}{|l|l|c|c|c|}
\hline \multicolumn{1}{|c|}{ Tên biến } & Ký hiệu & $\begin{array}{c}\text { Số biến } \\
\text { quan sát }\end{array}$ & $\begin{array}{c}\text { Cronbach's } \\
\text { alpha }\end{array}$ & $\begin{array}{c}\text { Hệ số } \\
\text { tương quan biến- } \\
\text { tổng nhỏ nhất }\end{array}$ \\
\hline Cảm nhận dễ sử dụng & CNDSD & 4 & 0,881 & 0,716 \\
\hline Hiẹ̉u quả mong đợi & HQMD & 5 & 0,896 & 0,700 \\
\hline Rủi ro trong giao dịch & RRGD & 5 & 0,838 & 0,550 \\
\hline Ảnh hưởng xã hội & AHXH & 4 & 0,821 & 0,574 \\
\hline Thương hiệu nân hàng & THNH & 4 & 0,756 & 0,505 \\
\hline Sự ưu thích cảm nhận & UTCN & 3 & 0,855 & 0,714 \\
\hline Quyết định sử dụng ngân hàng điện tử & QDSD & 4 & 0,782 & 0,568 \\
\hline
\end{tabular}

Đánh giá tính giá trị của thang đo

Bảng 3. Kết quả phân tích nhân tố khám phá biến độc lập

\begin{tabular}{|c|c|c|c|c|c|c|c|}
\hline & \multicolumn{6}{|c|}{ Hệ số tải nhân tố } & \multirow[t]{2}{*}{ Cấu trúc } \\
\hline & 1 & 2 & 3 & 4 & 5 & 6 & \\
\hline HQMD3 & 0,866 & & & & & & \multirow{5}{*}{$\begin{array}{l}\text { Hiệu quả } \\
\text { mong đọii }\end{array}$} \\
\hline HQMD1 & 0,853 & & & & & & \\
\hline HQMD2 & 0,832 & & & & & & \\
\hline HQMD4 & 0,831 & & & & & & \\
\hline HQMD5 & 0,808 & & & & & & \\
\hline RRGD4 & & 0,830 & & & & & \multirow{5}{*}{$\begin{array}{l}\text { Rủi ro trong } \\
\text { giao dịch }\end{array}$} \\
\hline RRGD3 & & 0,825 & & & & & \\
\hline RRGD1 & & 0,805 & & & & & \\
\hline RRGD5 & & 0,722 & & & & & \\
\hline RRGD2 & & 0,706 & & & & & \\
\hline CNDSD1 & & & 0,873 & & & & \multirow{4}{*}{$\begin{array}{c}\text { Cảm nhận dễ } \\
\text { sử dụng }\end{array}$} \\
\hline CNDSD3 & & & 0,854 & & & & \\
\hline CNDSD2 & & & 0,852 & & & & \\
\hline CNDSD4 & & & 0,841 & & & & \\
\hline AHXH2 & & & & 0,833 & & & \multirow{4}{*}{$\begin{array}{l}\text { Ảnh hưởng } \\
\text { xã hội }\end{array}$} \\
\hline AHXH4 & & & & 0,832 & & & \\
\hline AHXH3 & & & & 0,818 & & & \\
\hline AHXH1 & & & & 0,735 & & & \\
\hline UTCN3 & & & & & 0,891 & & \multirow{3}{*}{$\begin{array}{l}\text { Sự ưu thích } \\
\text { cảm nhận }\end{array}$} \\
\hline UTCN1 & & & & & 0,871 & & \\
\hline UTCN2 & & & & & 0,865 & & \\
\hline THNH2 & & & & & & 0,771 & \multirow{4}{*}{$\begin{array}{l}\text { Thương hiệu } \\
\text { ngân hàng }\end{array}$} \\
\hline THNH3 & & & & & & 0,763 & \\
\hline THNH5 & & & & & & 0,756 & \\
\hline THNH1 & & & & & & 0,743 & \\
\hline Eigenvalue & 3,67 & 3,329 & 2,963 & 2,624 & 2,368 & 2,054 & \\
\hline Phương sai trích \% & 14,679 & 13,316 & 11,853 & 10,497 & 9,474 & 8,217 & \\
\hline Phương sai tích lũy & 14,679 & 27,995 & 39,847 & 50,345 & 59,818 & 68,035 & \\
\hline & & Kiểm đị & $\begin{array}{l}\text { MO = }= \\
\text { Bartlett's }\end{array}$ & 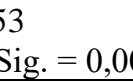 & & & \\
\hline
\end{tabular}

Phân tích nhân tố khám phá (EFA) được gọi là thích hợp khi $0,5 \leq \mathrm{KMO} \leq 1$. Hà Nam Khánh Giao và Bùi Nhất Vương (2019) cho rằng hệ số tải nhân tố $\geq 0,5$ được xem là có ý nghĩa thực tiễn. Kết quả Cronbach's Alpha cho thấy có 25 biến quan sát của 06 thành phần đo lường Quyết định sử dụng ngân hàng điện tử của khách hàng cá nhân đủ yêu cầu về độ tin cậy. Vì vậy, 25 biến quan sát của thang đo này được tiếp tục đánh giá bằng EFA. Kết quả EFA được trình bày 
tại bảng 3 .

Nhìn chung, tất cả các biến quan sát đều có hệ số tải nhân tố > 0,5 . Kiểm định KMO và Bartlett's trong phân tích nhân tố cho thấy sig. $=0,000<0,005$; hệ số KMO cao (bằng $0,753>$ $0,5)$. Kết quả này chỉ ra rằng các biến quan sát trong tổng thể có mối tương quan với nhau và phân tích nhân tố EFA rất thích hợp. Bên cạnh đó, tại các mức giá trị Eigenvalues lớn hơn 1 với phương sai trích là $68,035 \%$ (lớn hơn $50 \%$ ) đạt yêu cầu.

Bảng 4. Kết quả phân tích nhân tố biến phụ thuộc

\begin{tabular}{|l|lr|}
\hline & \multicolumn{1}{|c|}{ Hệ số nhân tố tải } \\
\cline { 2 - 3 } & \multicolumn{2}{c|}{} \\
\hline QDSD1 & & 0,798 \\
\hline QDSD4 & & 0,786 \\
\hline QDSD2 & & 0,767 \\
\hline QDSD3 & & 0,761 \\
\hline Eigenvalue & & 2,421 \\
\hline Phương sai trích tích lũy (\%) & \multicolumn{2}{c}{} \\
\hline \multicolumn{2}{|r|}{ KMO $=0,779$} \\
\hline
\end{tabular}

Kết quả phân tích EFA cho thấy phép trích được một nhân tố với 4 biến quan sát và phương sai trích tích lũy được là $60.532 \%(>50 \%)$. Hệ số $\mathrm{KMO}=0,779$ (giữa 0,5 và 1 ). Kết quả này chỉ ra rằng các biến quan sát trong tổng thể có mối tương quan với nhau và phân tích nhân tố (EFA) là thích hợp. Như vậy, sau khi phân tích nhân tố EFA ta thấy rằng mô hình lý thuyết ban đầu đề ra phù hợp với nghiên cứu. Các biến độc lập và biến phụ thuộc đã đạt được độ tin cậy và tính giá trị để sử dụng cho các phân tích tiếp theo.

\section{Phân tích tương quan}

Khi phân tích hồi quy, trước tiên là xem xét mối quan hệ tương quan tuyến tính giữa các biến độc lập với các biến phụ thuộc và giữa các biến độc lập với nhau. Ma trận tương quan ở bảng 5 trình bày các hệ số tương quan Pearson ( $\mathrm{r}$ ) giữa các biến nghiên cứu và mức ý nghĩa của từng hệ số đó. Mức ý nghĩa của các hệ số rất nhỏ $(\operatorname{sig}=0<0,05)$ nên các hệ số tương quan có ý nghĩa thống kê.

Bảng 5. Kết quả phân tích tương quan

\begin{tabular}{|c|c|c|c|c|c|c|c|c|}
\hline & & CNDSD & HQMD & RRGD & UTCN & AHXH & THNH & QDSD \\
\hline \multirow{3}{*}{ CNDSD } & Pearson Correlation & 1 &, $630^{* *}$ &,$- 604^{* *}$ &, $172^{\text {*** }}$ &,- 011 &, 061 &, $622^{* * *}$ \\
\hline & Sig. (2-tailed) & &, 000 &, 000 &, 001 &, 836 & ,263 &, 000 \\
\hline & $\mathrm{N}$ & 340 & 340 & 340 & 340 & 340 & 340 & 340 \\
\hline \multirow{3}{*}{ HQMD } & Pearson Correlation & $630^{* *}$ & 1 &,$- 570^{* *}$ & $145^{\text {** }}$ &,- 043 &,- 020 &, $665^{* * *}$ \\
\hline & Sig. (2-tailed) & ,000 & & , 000 &, 008 & ,432 & ,713 &, 000 \\
\hline & $\mathrm{N}$ & 340 & 340 & 340 & 340 & 340 & 340 & 340 \\
\hline \multirow{3}{*}{ RRGD } & Pearson Correlation &,$- 604^{* *}$ &,$- 570^{* *}$ & 1 &,- 080 &,- 011 &,- 098 &,$- 630^{\text {*** }}$ \\
\hline & Sig. (2-tailed) &, 000 & 000 & & 141 & 845 & ,071 &, 000 \\
\hline & $\mathrm{N}$ & 340 & 340 & 340 & 340 & 340 & 340 & 340 \\
\hline \multirow{3}{*}{ UTCN } & Pearson Correlation & $172^{* * *}$ & $145^{* * *}$ &,- 080 & 1 & $232^{* * *}$ &,$- 145^{* *}$ & $267^{* * *}$ \\
\hline & Sig. (2-tailed) &, 001 &, 008 & 141 & &, 000 &, 008 & , 000 \\
\hline & $\mathrm{N}$ & 340 & 340 & 340 & 340 & 340 & 340 & 340 \\
\hline \multirow{3}{*}{ AHXH } & Pearson Correlation &,- 011 &,- 043 &,- 011 & $232^{* * *}$ & 1 & 057 & $113^{*}$ \\
\hline & Sig. (2-tailed) & 836 & 432 &, 845 & ,000 & & 293 & ,037 \\
\hline & $\mathrm{N}$ & 340 & 340 & 340 & 340 & 340 & 340 & 340 \\
\hline \multirow{3}{*}{ THNH } & Pearson Correlation & ,061 &,- 020 &,- 098 &,$- 145^{* * *}$ & 057 & 1 & ,091 \\
\hline & Sig. (2-tailed) & 263 &, 713 &, 071 &, 008 & ,293 & & ,094 \\
\hline & $\mathrm{N}$ & 340 & 340 & 340 & 340 & 340 & 340 & 340 \\
\hline \multirow{3}{*}{ QDSD } & Pearson Correlation & $622^{* *}$ & $665^{* *}$ &,$- 630^{* * *}$ & $267^{* * *}$ &, $113^{*}$ & ,091 & 1 \\
\hline & Sig. (2-tailed) & ,000 & ,000 &, 000 &, 000 & 037 &, 094 & \\
\hline & $\mathrm{N}$ & 340 & 340 & 340 & 340 & 340 & 340 & 340 \\
\hline
\end{tabular}




\section{Phân tích hồi quy}

\section{Xem xét đa cộng tuyến}

Trong mô hình hồi quy đa biến, chúng ta giả thuyết các biến giải thích của mô hình không có hiện tượng đa cộng tuyến. Muốn kiểm định hiện tượng đa công tuyến ta xem xét hệ số phóng đại phương sai (VIF) và giá trị dung sai (Tolerance). Hệ số phóng đại phương sai ở bảng 6 của các biến đều nhỏ hơn 10 và giá trị dung sai của các biến đều bé hơn 2 cho thấy hiện tượng đa cộng tuyến rất thấp.

Bảng 7. Kết quả phân tích hồi quy

\begin{tabular}{|c|c|c|c|c|c|c|c|c|}
\hline & & Hệ số ch & chuẩn & $\begin{array}{c}\text { Hệ số } \\
\text { chuẩn hóa }\end{array}$ & & & Hệ số đa cế & tuyến \\
\hline & Mố hình & B & $\begin{array}{l}\text { Sai số } \\
\text { chuẩn }\end{array}$ & Beta & $\mathbf{t}$ & Sig. & Tolerance & VIF \\
\hline & (Hằng số) & 1,680 & 0,357 & & 4,710 & 0,000 & 0,505 & 1,981 \\
\hline & CNDSD & 0,213 & 0,055 & 0,188 & 3,845 & 0,000 & 0,538 & 1,860 \\
\hline & HQMD & 0,393 & 0,051 & 0,367 & 7,750 & 0,000 & 0,568 & 1,761 \\
\hline 1 & RRGD & $-0,314$ & 0,051 & $-0,287$ & $-6,219$ & 0,000 & 0,884 & 1,132 \\
\hline & UTCN & 0,145 & 0,036 & 0,149 & 4,036 & 0,000 & 0,930 & 1,075 \\
\hline & $\mathrm{AHXH}$ & 0,141 & 0,057 & 0,089 & 2,469 & 0,014 & 0,949 & 1,053 \\
\hline & THNH & 0,048 & 0,023 & 0,075 & 2,111 & 0,036 & 0,505 & 1,981 \\
\hline & ến phụ thuộ & $\overline{\mathrm{DSD}}$ & & & & & & \\
\hline & hiệu chỉnh: & & & & & & & \\
\hline & ống kê F (Al & VA): 8 & & & & & & \\
\hline & c ý nghĩa (S & - $A J$ & A): 0 , & & & & & \\
\hline
\end{tabular}

Bảng 6 cũng cho thấy $\mathrm{R}^{2}$ hiệu chỉnh bằng 0,591 có nghĩa là $59,1 \%$ sự biến thiên của QDSD (Quyết định sử dụng ngân hàng điện tử) được giải thích bởi sự biến thiên của 06 biến độc lập THNH, HQMD, AHXH, UTCN, RRGD, CNDSD. Ngoài ra, trị số F có mức ý nghĩa với Sig. $=0,000(<0,05)$ có nghĩa mô hình hồi quy tuyến tính đưa ra là phù hợp với dữ liệu thực tế thu thập được và các biến đưa vào đều có ý nghĩa trong thống kê với mức ý nghĩa $5 \%$.

Phương trình hồi quy chưa chuẩn hóa có dạng:

$$
\begin{gathered}
\mathrm{QDSD}=1,680+0,213 * \mathrm{CNDSD}+0,393 * \mathrm{HQMD}-0,314 * \mathrm{RRGD}+0,141 * \mathrm{AHXH}+ \\
0,048 * \mathrm{THNH}+0,145 * \mathrm{UTCN}
\end{gathered}
$$

Kết luận: so sánh mức độ tác động của 06 biến này vào biến phụ thuộc Quyết định sử dụng dịch vụ ngân hàng điện tử theo thứ tự giảm dần như sau: Hiệu quả mong đợi có tác động mạnh nhất $\left(\beta_{2}=0,367\right)$; Rủi ro trong giao dịch $\left(\beta_{3}=-0,287\right)$; Cảm nhận dễ sử dụng $\left(\beta_{1}=0,188\right)$; Sự ưa thích cảm nhận $\left(\beta_{6}=0,149\right)$; Ảnh hưởng xã hội $\left(\beta_{5}=0,089\right)$, và tác động thấp nhất là biến Thương hiệu ngân hàng $\left(\beta_{4}=0,075\right)$. Như vậy các giả thuyết $\mathrm{H}_{1}, \mathrm{H}_{2}, \mathrm{H}_{3}, \mathrm{H}_{4}, \mathrm{H}_{5}, \mathrm{H}_{6}$ đều được chấp nhận ở độ tin cậy $95 \%$.

\section{HÀM Ý QUẢN TRI}

\section{Đối với yết tố "Hiệu quả mong đọii"}

Yếu tố Hiệu quả mong đợi là yếu tố tác động mạnh nhất đến quyết định sử dụng dịch vụ ngân hàng điện tử của khách hàng cá nhân tại Ngân hàng Agribank Cần Thơ. Vì vậy, Ngân hàng Agribank cần phải cung cấp dịch vụ và hỗ trợ giúp khách hàng cảm thấy thuận tiện khi giao dịch, giúp khách hàng cảm nhận được hữu ích khi sử dụng dịch vụ. Ngân hàng cần có đường dây hỗ trợ dịch vụ khách hàng khi khách hàng có vướng mắc, giải quyết khiếu nại cho khách hàng nhanh chóng. Đồng thời Agribank cần phải đẩy mạnh hoạt động dịch vụ marketing cho 
dịch vụ ngân hàng điện tử, đào tạo nhân viên tiếp thị chuyên nghiệp. Bên cạnh đó, Agribank nên cung cấp các dịch vụ ở mức độ cao hơn và mang lại nhiều lợi nhuận hơn như dịch vụ quản lý quỹ, cho thuê tài chính, điện tử hóa các thủ tục,...tiến tới xây dựng các chi nhánh hoạt động trên môi trường điện tử.

\section{Đối với yếu tố "Rủi ro trong giao dịch"}

Theo kết quả nghiên cứu, vấn đề rủi ro giao dịch có ảnh hưởng mạnh thứ hai đến quyết định sử dụng dịch vụ ngân hàng điện tử. Khi xây dựng hệ thống ngân hàng điện tử, ngân hàng nên quan tâm đến vấn đề bảo mật và toàn vẹn dữ liệu, hạn chế những rủi ro phát sinh. Các sản phẩm e-banking luôn tiềm tàng rủi ro giao dịch cao, khách hàng giao dịch qua kênh này thường ít kiên nhẫn với những thiểu sót của ngân hàng. Để nâng cao mức độ an toàn cho các giao dịch ngân hàng Agribank cần quản lý chặt chẽ quá trình triền khai và kiểm tra hệ thống. Cung cấp các thông tin về hệ thống của ngân hàng: cần hướng nhân viên hướng dẫn khách hàng cụ thể và rõ ràng về các rủi ro và lợi ích của khách hàng khi cung cấp dịch vụ cho khách hàng, cần thông báo đầy đủ và chính xác cho khách hàng về quyền lợi, nghĩa vụ và trách nhiệm của khách hàng và của ngân hàng đối với các vấn đề liên quan đến các giao dịch trực tuyến, đặc biệt là những vấn đề có thế phát sinh từ những lỗi xử lý và vi phạm an ninh hệ thống.

\section{Đối với yếu tố "Cảm nhận dễ sử dụng”}

Yếu tố Dễ sử dụng là yếu tố tác động mạnh thứ 3 đến đến quyết định sử dụng dịch vụ ngân hàng điện tử. Hiện tại, dịch vụ ngân hàng điện tử được cung cấp bởi rất nhiều ngân hàng, mỗi ngân hàng đều cố gắng hoàn thiện dịch vụ để mang lại lợi ích cho khách hàng cao nhất. Trong bối cảnh khó khăn và cạnh tranh đó, Ngân hàng Agribank nên cố gắng hoàn thiện dịch vụ, đưa ra các phiên bản dễ dàng sử dụng trên máy tính, và điện thoại, các tính năng ngày càng nhiều, tiện ích cao đồng thời giao diện dễ sử dụng. Agribank nên phát hành cẩm nang giao dịch trên Agribank Online để khách hàng có thể dễ dàng sử dụng hơn. Hiển thị số điện thoại hỗ trợ trên màn hình dịch vụ e-banking, hiện nay, số điện thoại hỗ trợ cho cả hệ thống của Ngân hàng Agribank chuyên về dịch vụ ngân hàng điện tử luôn trong tình trạng quá tải. Hơn nữa, Agribank nên hoàn thiện và thiết kế website cho dễ sử dụng, thông tin chặt chẽ, phong phú, đầy đủ và được cập nhật liên tục. Từ ngữ được sử dụng phải rõ ràng, dễ hiểu giúp khách hàng nắm bắt được những thông tin thiết yểu trong thời gian ngắn nhất.

\section{Đối với yếu tố "Sự ưa thích cảm nhận"}

Yếu tố Sự ưa thích cảm nhận là yếu tố tác động mạnh thứ 4 đến đến quyết định sử dụng dịch vụ ngân hàng điện tử. Ngân hàng Agribank cần thiết kế vị trí hiển thị các ứng dụng tiện ích của internet banking một cách khoa học. Agribank cần nghiên cứu thiết kế vị trí các tính năng khách hàng thường ưu tiên sử dụng sao cho trực quan, dễ truy cập hơn, qua đó, giúp khách hàng tiết kiệm thời gian trong việc sử dụng các tính năng với tần suất sử dụng cao cũng như đỡ mất công tìm kiếm các tính năng cần thiết. Từ đó, sẽ nâng cao được sự thích thú sử dụng dịch vụ của khách hàng cá nhân. Ngoài ra, bên cạnh các tiện ích thanh toán tiền điện nước, vé máy bay, nạp tiền điện thoại..., Agribank cần chủ động mở rộng kết nối với hệ thống các đơn vị bán hàng trực tuyến, đặc biệt là các hệ thống cung cấp các hàng hoá thông thường nhằm cung ứng dịch vụ mua hàng trực tuyến và thanh toán qua điện thoại di động cho người tiêu dùng. Điều này sẽ định hướng và dẫn dắt khách hàng sử dụng dịch vụ mobile banking trong các giao dịch kinh doanh và giúp internet banking đi vào cuộc sống hàng ngày.

\section{Đối với yếu tố "Ảnh hưởng xã hội”}

Ảnh hưởng xã hội có tác động thứ năm đến quyết định sử dụng dịch vụ ngân hàng điện tử. Ngày nay, với sự ra đời và phổ biến của các thiết bị, công nghệ mới như điện thoại di động, internet, truyền hình ...dần dần chi phối thói quen sinh hoạt của những người dân. Không chỉ đóng vai trò là thiết bị thông tin, liên lạc mà thiết bị này còn là phương tiện giúp người dân 
quản lý cuộc sống, học tập, trao đổi kiến thức, kinh doanh thương mại, vui chơi giải trí .... Với việc cung cấp dịch vụ tốt, những hỗ trợ trực tuyến tốt cũng là một cách đem lại ấn tượng tốt trong lòng khách hàng khi giao dịch. Vì vậy, ban lãnh đạo ngân hàng có thể thông qua các tổ chức, cá nhân trên để giới thiệu, quảng bá loại hình dịch vụ ngân hàng điện tử đến với nhiều người tiêu dùng tiềm năng, làm cho khách hàng trở nên gần gũi, quen thuộc với khái niệm này. Bên cạnh đó, ngân hàng cần phải nâng cao chất lượng dịch vụ, hỗ trợ giải đáp những vướng mắc cũng như các vấn đề mà khách hàng gặp phải trong quá trình sử dụng, giúp khách hàng có được sự an tâm trong việc sử dụng dịch vụ điện tử được ngân hàng cung cấp, trở thành cầu nối để giới thiệu với gia đình, người thân và bạn bè.

\section{Đối với yếu tố "Thương hiệu ngân hàng"}

Hình ảnh ngân hàng có tác động yếu nhất đến quyết định sử dụng dịch vụ ngân hàng điện tử.Vì vậy để tiếp tục giữ vững và nâng cao hình ảnh của mình, Agribank cần phải tiếp tục xây dựng và nâng cao hình ảnh, thương hiệu Agribank bằng cách giữ vững và thực hiện thành công các mục tiêu kinh doanh đã đề ra. Cần triển khai các sản phẩm dành cho khách hàng cá nhân đa dạng, phong phú, gần gũi với người sử dụng nhằm tạo cho họ có ấn tượng tốt đối với Agribank. Tiếp tục mở rộng mạng lưới hoạt động kinh doanh (chi nhánh, phòng giao dịch) đảm bảo phủ kín các quận, huyện tại địa bàn Cần Thơ nói riêng và các địa bàn trọng tâm trong cả nước. Tiếp tục thực hiện công tác truyền thông, quảng bá thương hiệu Agribank trên các phương tiện thông tin truyền thống, đặc biệt là việc quảng bá hình ảnh Agribank trên các đài truyền hình địa phương. Các chương trình quảng bá phải phù hợp với văn hóa và thói quen từng vùng miền để đạt được hiệu quả cao.

\section{HẠN CHẾ CỦA ĐỀ TÀI VÀ HƯỚNG NGHIÊN CÚU TIẾP}

Thứ nhất, nghiên cứu này chỉ giải thích được $59,1 \%$ sự biến thiên của quyết định sử dụng dịch vụ ngân hàng điện tử của khách hàng cá nhân tại Ngân hàng Agribank Cần Thơ. Do đó, vẫn còn một số yếu tố khác ảnh hưởng đển quyết định lựa chọn của khách hàng chưa được đề cập trong nghiên cứu này. Thứ hai, nghiên cứu này chỉ được thực hiện tại Ngân hàng Agribank Cần Thơ. Khả năng khái quát của mô hình sẽ cao hơn nếu nghiên cứu được lặp lại trên nhiều địa phương khác. Thứ ba, nghiên cứu tiếp theo nên tiếp tục phỏng vấn thêm nhiều khách hàng khác nhau ở các phân khúc khác nhau đề đưa ra những đánh giá cụ thể và chính xác hơn về ảnh hưởng của các nhân tố đến quyết định sử dụng ngân hàng điện tử của khách hàng.

\section{TÀI LIỆU THAM KHẢO}

Ajzen, I. (1980). From intentions to action: a theory of planned behavior. Berlin and New York: Springer-Verlag.

Ajzen, I. (1991). The theory of planned behavior. Organizational Behavior and Human Decision Processes, 50(2), 179-211.

Burns, A. C., \& Bush, R. F. (1995). Marketing Research. New Jersey: Prentice Hall.

Davis et al. (1989). User acceptance of computer technology: A comparison of two theoretical models, Management Science, 35, 982-1003.

Fatimah, F. O., \& Suyanto, A. (2016). Analysis Factors of Using Internet Banking in Indonesia, Management and Organizational Studies, 3(2), 10-15.

Fishbein, M., \& Ajzen, I. (1975). Belief, attitude, intention, and behavior. MA: AddisonWesley.

Giao, H. N. K., \& Vuong, B. N. (2019). Giáo trình cao học phưong pháp nghiên cúu khoa học trong kinh doanh cập nhật SmartPLS. Hồ Chí Minh: Nhà Xuất Bản Tài Chính.

Jayawardhena C., \& Foley P. (2000). Changes in the banking sector-the case of internet banking in the UK, Internet Research: Electronic Networking Applications and Policy, 10(1), 19-30. 
Maditinos et al. (2013). An examination of the critical factors affecting consumer acceptance of online banking A focus on the dimensions of risk, Journal of Systems and Information Technology, 15(1), 97-116.

Venkatesh, V., \& Davis, F. D. (2000). A theoretical extension of the technology acceptance model: Four longitudinal field studies. Management Science, 46, 186-204.

Venkatesh et al. (2003). User Acceptance of Information Technology: Toward a Unified View, MIS Quarterly, 27(3), 425-478. 